\title{
Les classifications du vieillissement :
}

\author{
Quelle résonnance dans les représentations \\ des femmes âgées?
}

\author{
Isabelle Marchand \\ Anne Quéniart \\ Michèle Charpentier ${ }^{1}$
}

\begin{abstract}
[Résumé] L'objectif de cet article vise à confronter les classifications savantes relatives à la vieillesse aux représentations sociales du vieillissement de femmes âgées de 65 ans et plus. En utilisant la théorie des classifications interactives de Hacking (2006), nous chercherons à cerner de quelle manière les classifications de l'âge, de l'activité et de la fragilité résonne dans les représentations du vieillissement de femmes aînées, interrogées dans le cadre d'une récente recherche qualitative. Les interactions entre ces deux dimensions catégorielles s'expriment en termes de résistance face à la catégorie "femmes aînées/âgées ». La notion de l'activité est utilisée tant pour réfuter la vieillesse que pour assurer cette «continuité de soi » et son engagement dans la vie malgré le processus de fragilisation vécu.

Mots-clés : femmes aînées/âgées, vieillissement, vieillesse, activité, fragilité

[Abstract] This article aims to challenge the erudite classifications regarding aging and the social representations of aging in women aged 65 years and older. Using Hacking's (2006) theory of interactive classifications, we attempt to identify the ways in which classifications of age, activity, and fragility resonate with the representations of aging from the perspectives of older women. These women participated in interviews in the context of a recent qualitative study. The interactions between these two dimensions were expressed in terms of resistance to the category of "elderly woman". The notion of activity was used for refuting aging as well as to ensure a "continuity of self" and one's involvement in life activities despite the lived process of increased frailty.

Keywords: older/elderly women; aging, age, activity, frailty
\end{abstract}

T a fixation d'un seuil d'âge à partir duquel la vieillesse commence est une manière ـancienne et commune de quantifier le vieillissement démographique. Si en France ce seuil de la vieillisse a été établi à 60 ans en 1928 sous la plume de Sauvy, il varie aujourd'hui selon les pays et les instances politiques, le rendant par là même tributaire

1 Isabelle Marchand est candidate au doctorat en service social, Université de MontréalMcGill (Québec, Canada). Anne Quéniart est professeure titulaire au Département de sociologie de I'Université du Québec à Montréal (Québec, Canada). Michèle Charpentier est professeure titulaire à l'École de service social de l'Université du Québec à Montréal (Québec, Canada). 
des contextes sociopolitiques (Bourdelais, 1993). Instrument d'intervention de l'État, cette frontière qui marque le « devenir vieux » contribuera aussi à la stéréotypie de la vieillesse, qui sera longtemps associée à la déchéance durant le XXe siècle (Ibid). Ces représentations négatives, qui ont cristallisé la vieillesse comme un problème social, joueront un rôle fondamental dans le développement de politiques de la vieillesse d'après-guerre (Guillemard, 1984). L'émergence de représentations entourant la vieillesse "épanouie», qui vient faire contre poids à la vieillesse jusqu'ici "ingrate» (Hummel, 2001), transformera à son tour l'intervention de l'État et ses politiques face aux nouvelles réalités du vieillissement.

Dans le cadre de cet article, nous nous intéresserons à cette interaction entre les instruments de la gouvernance publique, plus particulièrement aux grilles de classification utilisées en matière de vieillissement, et le discours de femmes aînées à propos de leur vieillissement. Plus spécifiquement, nous confronterons des classifications savantes relatives à la vieillesse aux représentations sociales du vieillissement de femmes âgées de 65 ans et plus. Nous présenterons d'abord les classifications mobilisées en matière d'âge, d'activité et de fragilité/dépendance. Nous résumerons ensuite la méthodologie de la recherche qualitative de laquelle sont tirées les représentations des femmes aînées. Par la suite seront exposés les résultats de la confrontation entre les trois classifications retenues et les représentations des répondantes, puis nous discuterons de leurs aspects interactifs : c'est-à-dire, de quelles manières les perceptions du vieillir interpellent-elles ces classifications et comment, à leur tour, ces dernières interagissent-elles ou influencent-elles les répondantes? Nous conclurons en soulevant quelques enjeux globaux concernant les rapports de sexe et d'âge.

\section{L'utilisation de classifications dans la vieillesse : l'âge, l'activité et la dépendance}

La catégorie « aînée/personne âgée », définie à partir de 65 ans par les mesures statistiques au Canada, est la première classification que nous retenons puisqu'elle représente le seuil d'entrée dans la vieillesse, donnant accès aux mesures d'aide financière qui y sont associées (pension de la sécurité de la vieillesse, rentes). La deuxième classification concerne l'activité dans le vieillissement, dimension qui est depuis longtemps considérée comme importante, car elle permet le maintien du bien-être et de la qualité de vie dans la vieillesse (Bickel, 2007). Parmi d'autres études, les travaux de Bickel (2007) opérationnalisent l'activité au moyen d'une liste qui en répertorie seize (annexe 1), tant d'intérieures (lectures, télévision, etc.) que d'extérieures (loisirs, sorties culturelles, etc.). Nous retendrions donc cette grille puisque l'activité y est définie en dehors du travail rémunéré et, ce faisant, prend en compte plusieurs types d'activités accomplies dans la vie quotidienne.

La dernière classification a trait au concept de la dépendance, définie comme «l'état de la personne âgée qui ne peut accomplir seule les activités de la vie courante» 
(Ylieff et al., 2006: 1). Divers outils standardisés ont été élaborés pour l'évaluer (grille de Colvez, l'échelle de Katz, la grille AGGIR, etc.) (Ibid). En continuité avec la classification de l'activité retenue, nous privilégierons la classification de Bickel (2007) (annexe 2) puisqu'elle introduit à la fois la notion de fragilité et celle de la dépendance/indépendance, que l'auteur mesure en regard de la grille de Katz (celle-ci évaluant les capacités des personnes âgées à accomplir les activités nécessaires au quotidien, annexe 3). Plus spécifiquement, l'auteur propose une opérationnalisation de la fragilité à l'aide de cinq dimensions de la santé, étayées par dix-neuf indicateurs. En regard de cette échelle, trois statuts de santé sont définis : 1) une personne est fragile (affectée par au moins deux des cinq dimensions de la santé sans aucune incapacité fonctionnelle, mesurée avec l'échelle de Katz sur les activités quotidiennes) ; 2) est dépendante (aux prises avec au moins une de ces incapacités); ou 3) est indépendante (touchée par aucune des dimensions de la fragilité ou incapacités définies dans la grille de Katz).

\section{Précisions méthodologiques}

Afin de mettre ces classifications à l'épreuve du réel, nous les avons confrontées aux résultats d'une récente recherche qualitative que nous avons menée sur les représentations sociales des femmes aînées quant au vieillissement2. Considérant la pertinence de poursuivre la recherche empirique sur les groupes sociaux historiquement minorisés afin de mieux connaître les expériences multiples et complexes du vieillir, et ce, au regard d'un contexte sociohistorique et culturel donné, nous nous sommes ici intéressées aux femmes âgées nées au Québec et d'origine linguistique francophone. En dépit d'un intérêt grandissant depuis les années 1980, rappelons que la vieillesse au féminin reste un champ d'études peu investigué, notamment dans la francophonie, ce y compris au sein des études féministes ou de genre (Charpentier et Billette, 2010; Quéniart et al, 2011). À partir d'une perspective critique du vieillissement (Minkler, 2007), notre recherche a ainsi cherché à apporter un éclairage sur la diversité des expériences des femmes âgées québécoises, en mettant en lumière : sur le plan macro, les dynamiques relatives aux rapports sociaux qui traversent et modèlent leur vieillissement: sur le plan micro, l'interprétation que font les actrices de leurs expériences du vieillir à partir de leurs trajectoires.

À partir d'un échantillonnage théorique (Paillé, 1994), vingt-cinq aînées ont été recrutées dans divers milieux de vie. Elles sont issues de trois cohortes d'âges : 9 répondantes sont âgées de 65-74 ans (le 3e âge), 10 de 75-84 (le 4e âge) et finalement, 6

2 Les femmes aînées dans l'espace public et privé : quels héritages, legs, transmission?, sous la direction de Michèle Charpentier et Anne Quéniart (Conseil de recherche de sciences humaines du Canada, 2008-2011). 
femmes sont âgées de 85 ans et plus (5e âge). Sur le plan socio-économique, 8 femmes disposent de revenus modestes à faibles, 14 se situent dans la classe moyenne et 3 vivent au sein de milieux sociaux plus nantis (classe moyenne supérieure). La majorité des femmes sont soit mariées (9), soit veuves (9), et les autres sont célibataires (3) et divorcées ou séparées (4). Sur le plan de la formation scolaire, 8 des 9 femmes de la première génération (65-74 ans) détiennent un diplôme postsecondaire. Au sein de cette cohorte, toutes ont occupé un emploi rémunéré en ayant une famille (qu'elles soient mariées ou séparées/divorcées). Les femmes âgées de plus 75 ans partagent pour la plupart un parcours traditionnel (i.e travail non rémunéré dans le domaine privé après le mariage). Des entrevues semi-dirigées en profondeur ont été réalisées. Les thèmes abordés au cours de ces entretiens s'articulaient autour de deux dimensions : d'une part, les représentations des femmes aînées face au vieillissement, leurs places et leurs rôles dans la société, leur rapport au bien vieillir ; d'autre part, la dynamique de la transmission intergénérationnelle (valeurs et savoirs transmis, modes et sens de la transmission). La présente contribution mobilise les résultats de la première dimension, en raison de leur résonnance avec les classifications retenues.

Toutes les entrevues ont été retranscrites et codées afin de générer des thèmes (anticipés et émergents). Dans une démarche résolument inductive s'inspirant de la théorisation ancrée (Paillé, 1994), une analyse de contenu a été réalisée afin d'objectiver l'évidence immédiate du matériau, et d'étudier les contextes (macro et micro) entourant la production des discours. À partir des thèmes générés, différentes catégories conceptuelles ont été créées et raffinées dans un processus itératif entre les thèmes issus du matériau et les catégories. Mises ensuite en relation les unes avec les autres, des hypothèses interprétatives ont été élaborées et confrontées à des théories explicatives globales.

\section{Représentations du vieillir de femmes âgées : quel écho avec les classifications de la vieillesse?}

L'analyse des entretiens nous a permis de dégager les rapports des répondantes au vieillissement (leur réalité discursive dans l'ici et maintenant face au vieillissement), mais aussi le regard qu'elles portent sur elles-mêmes en tant que femmes vieillissantes à la lumière de leurs parcours et de leurs conditions de vie. Les résultats obtenus de cette recherche, lorsque nous les confronterons aux trois classifications décrites précédemment, nous permettront alors de réfléchir aux questions suivantes : de quelles manières leurs perceptions du vieillir interpellent-elles ces classifications et comment, à leur tour, ces classifications interagissent ou influencent-elles les répondantes? 


\section{1 . Tensions et résistance face à la catégorie « aînées "}

Une majorité des femmes aînées rencontrées (soit 21), toutes catégories d'âges et profils confondus, refusent d'être considérées comme des femmes aînées ou, du moins, ne se « voient » pas comme appartenant à cette catégorie d'âge associée aux 65 ans et plus. Dans le même ordre d'idée, plusieurs répondantes mentionnent ne pas «se sentir vieilles » ou « comme une femme aînée » lorsque nous leur demandons «Qu'est-ce qu'une femme âgée pour vous? » :

On se sent pas aînées tant que ça, on vit notre vie, puis je me sens pas cet âgelà. [...] Moi, je ne m'engage pas dans des cercles [d'aînés]. J'ai de la misère avec ça. (Denise, 85 ans)

Je suis peut être pas correcte, mais il me semble que je me considère pas encore comme une femme aînée (Aline, 77 ans).

Une femme aînée, c'est quelqu'un de vieille. Moi, dans ma tête, j'ai toujours 19 ans ! » (Barbara, 67 ans)

Dans le même registre, pour d'autres répondantes, la catégorie « aînée " renvoie à l'image d'une « vieille femme » cloîtrée, vivant en marge de la société. Plus encore, les appellations «femme âgée », " aînée », expriment des représentations négatives ayant trait à la maladie, aux incapacités et au fait d'habiter dans des centres d'hébergement pour les personnes âgées qui incarnent l'enfermement et l'exclusion sociale.

Q : Qu'est-ce qui vous vient en tête quand on parle des femmes aînées ou âgées?

[Les femmes aînées], elles vont faire du bingo, elles font un petit peu de ceci, de cela. Elles sont assises derrière une fenêtre, puis elles attendent, elles sont aussi dans les centres d'hébergement, c'est comme une prison aussi. (Laure, 65 ans)

Ils sont assis sur une chaise [les aînés], puis [ils] attendent que quelqu'un viennent les voir [...], sinon, ils sont malades, pour moi, c'est ça, une vraie aînée, qui ne peut pas avancer rien. (Jeanine, 70 ans)

Par ailleurs, d'autres représentations émergent lorsque quatre répondantes parlent de leurs contemporaines, une génération de femmes nées après la Première Guerre mondiale, ayant connu les grandes crises économiques et les profondes mutations sociales qui ont accompagné les Trente Glorieuses. En évoquant « leur génération », elles désignent des femmes d'une même cohorte d'âges, nées dans un contexte sociohistorique précis, avec lesquelles dont elles partagent des référents familiers et une certaine vision du monde, basée sur des «évènements fondateurs » qui les distinguent d'autres générations (Mauger, 2009). Leurs représentations se basent sur une gamme d'attributs qui expriment une vision positive d'être devenue des femmes âgées : maturité, intelligence, résilience face aux épreuves, etc., comme l’illustre le témoignage de Chantal (80 ans).

Q : Quelle image vous vient en tête quand on parle des femmes aînées? 
Ce sont des femmes de ma génération en général, des femmes assez intelligentes qui ont vécu les mêmes choses que moi là, les difficultés, à la même époque que moi. On n'en revient pas d'être rendu là où on est rendu [comme société]. Chantal (80 ans)

Pour ces dernières, appartenir à la catégorie "femme aînée » exprime ainsi une «proximité identitaire collective» (Hummel, 2001), mais ici construite sur l'idée d'appartenance à une même génération sociale (Mauger, 2009). Cependant, la plupart des répondantes (soit 21) résistent à cette classification, d'une part, avec une "proximité identitaire individuelle» (« moi » « je ») (Hummel, 2001), pour se dissocier des â̂nées vraiment «vieilles» ou «malade». Différentes stratégies sont déployées pour mettre à distance cette appartenance catégorielle « aînée/vieille »; elles s'expriment notamment par la mobilisation des notions de l'activité et de l'autonomie.

\subsection{Rester active pour subvertir la vieillesse}

À la question «Qu'est-ce que c'est pour vous vieillir? », les témoignages mettent l'accent sur l'importance de rester active, au courant de ce qui se passe dans la société, de maintenir un esprit alerte et éveillé sur le monde. Pour les répondantes, cette attitude permet d'échapper au recroquevillement sur soi-même et à la taciturnité, autres représentations négatives associées à la vieillesse.

Vieillir, c'est arrêter d'avoir des projets. Autour de moi toutes les personnes qui s'appellent des «vieilles», c'est des personnes qui n'ont plus de projets. Quand on reste replié sur soi, là on vieillit. (Adèle, 73 ans)

On vieillit quand l'esprit se referme sur lui-même, c'est là qu'on devient toute ratatinée. [...]. Pour moi, c'est important de rester ouvert aux autres personnes et à ce qui se passe dans le monde, il faut rester active intellectuellement. (Claire, 72 ans)

Pour une autre répondante, être active s'actualise dans le maintien de ses activités militantes au sein du mouvement des femmes. À 71 ans, Josée insiste ainsi sur le « devoir de citoyenne » que les femmes âgées ont à jouer au sein de la société. Discours similaire pour Odette, qui continue à faire du bénévolat dans l'organisme communautaire dans lequel elle a longtemps travaillé avant de prendre sa retraite.

$\mathrm{Q}$ : Et puis les femmes de votre génération, comment voyez-vous leur place et leur rôle dans notre société, autour de vous?

J'imagine mal qu'à 65 ans et plus, des femmes vivent repliées sur elles-mêmes, que la seule chose ou les seuls intérêts qu'elles ont c'est d'aller jouer aux cartes, au bingo ou de s'organiser des voyages. J'ai de la difficulté à concevoir qu'on peut vivre seulement en tournant autour de soi même, et ça à tous les âges. (Josée, 70 ans)

Je suis passionnée par mes activités de bénévolat, je sais que c'est utile, puis ça me passionne d'avoir des projets comme ça, alors j'en vois des femmes autour 
de moi qui ont mon âge et qui continuent de faire des choses intéressantes. (Odette, 73 ans)

À la question «Et bien vieillir, qu'est-ce que cela veut dire selon vous? », elles sont plusieurs (soit 23) à évoquer l'importance d'être active régulièrement, de veiller à son apparence physique, de maintenir une bonne condition physique ainsi qu'une vie sociale. Nulle question d'associer leur vieillesse au ralentissement, à l'inactivité, tant sociale que physique et psychologique :

Des femmes de mon âge qui aiment encore se pomponner, aller à la coiffeuse, se faire faire les ongles, aller magasiner, sortir, prendre un verre de vin avec des amies. (Jeanine, 70 ans)

Il faut continuer à faire des exercices, et toutes sortes d'affaires pour se garder en forme physiquement et moralement, faut prendre soin de nous-mêmes pour bien vieillir. (Lise, 93 ans)

Pour les répondantes, rester active réfère à une série d'activités opérationnalisées par Bickel (2007), soit tant les activités extérieures (visiter des amies, les activités culturelles et de loisirs) que celles ayant trait aux activités physiques et manuelles. D'autres activités quotidiennes telles que la consommation de médias ou de lecture sont aussi mobilisées par les répondantes dans une visée de maintien de l'acuité intellectuelle et surtout, au fur et à mesure de l'avance en âge, de « rester dans la vie » et non en marge du temps et des réalités contemporaines. En évoquant diverses études qualitatives sur l'activité dans la vieillesse, Boudiny (2012:12, traduction libre) précise que ces activités « ordinaires » et quotidiennes sont un « indicateur plus important de l'engagement dans la vie sociale que plusieurs activités sociales et physiques associées à la jeunesse ». Les activités du quotidien mettent ainsi en relief une dimension relationnelle importante : le rapport aux autres (ne pas être ou se sentir isolé), au monde (garder un sentiment de familiarité vis-à-vis de celui-ci) (Caradec, 2007) et à soi-même (maintenir du bien-être).

\subsection{Fragilité, dépendance et indépendance dans la vieillesse}

Au regard des trois statuts de santé dans la vieillesse définis par Bickel (2007), soit la fragilité, la dépendance et l'indépendance, les propos des répondantes âgées de 75 ans et plus font en majorité écho à la fragilité (soit 15 sur 16), parfois pour parler de leur rapport au vieillissement et tantôt pour expliquer comment elles négocient le maintien de certaines activités et la représentation de soi dans la grande vieillesse. Dans cette perspective, deux nonagénaires mobilisent toujours la classification d'être active. Étant cependant confrontées au processus de la fragilisation (i.e la perte de mobilité correspond au devenir fragile dans la classification de Bickel, 2007), elles doivent adapter ou changer les types d'activités réalisées.

$\mathrm{Q}$ : Et bien vieillir, qu'est-ce que cela veut dire selon vous? 
Il faut continuer à s'intéresser à ce qui se passe dans l'actualité, pas se tenir éloigné. [....] Moi je suis un peu spéciale de ce côté-là, je marche lentement, mais je lis, j'écoute la télé... (Arlette, 98 ans)

Je continue à faire exactement ce que je faisais, mais plus lentement, différemment, je m'adapte...» (Gabrielle, 94 ans)

Je ne danse plus comme avant parce que, il y a deux ans, j'ai fait un léger arrêt cardiaque et j'ai appris à me calmer le pompom [...]. Quand je suis fatiguée, je prends ça moderato, mais reste indépendante dans mes affaires. (Rita, 81 ans)

Pour les répondantes ayant fait allusion au processus de fragilisation en cours, la classification de l'activité retenue interagit avec celle de la fragilité. Si le maintien ou la réorganisation dans le temps des activités sociales, physiques et quotidiennes permet de maintenir une position d'inclusion dans le monde social (Lavigne d'Épinay et Spini, 2008), elle est aussi énoncée pour contrer le «ressenti » de la vieillesse. De la même façon, la classification de la fragilité/dépendance interagit avec celle de l'âge. Josette (91 ans), ayant une mobilité décroissante au cours des années, ne s'identifie pas à une aînée: "Il n'y en a pas beaucoup de vieilles madames. Elles ont une marchette ${ }^{3}$, ça, c'est correct, mais de bien malades, là, non, je ne pourrais pas dire ça ». Ajoutant qu'elle est «toujours debout», elle peut ainsi préserver une certaine autonomie dans l'accomplissement de ses besoins quotidiens (telle que définie dans la grille de Katz (1970, dans Bickel, 2007). La résistance est ici manifeste quant au fait de se définir par l'amalgame "vieille-malade», mais étant trop fragilisée pour évoquer l'activité comme stratégie permettant de mettre à distance la vieillesse, elle mobilise la notion d'autonomie (car elle n'est pas encore dépendante au sens de Katz).

$\mathrm{Au}$ fur et à mesure que le processus de fragilisation suit son cours, le sentiment de finitude se fait prégnant dans le discours de Thérèse (86 ans), qui prend pleinement conscience de cette dernière étape de vie en raison de son état de dépendance : « on va mourir un jour, je suis en chaise roulante [...]. Pour les choses de tous les jours, je suis pu capable de faire grand-chose toute seule, alors ce n'est pas vivre ça. Mais en autant que je me voie différemment, c'est vivre. En l'occurrence, cette répondante en état de dépendance oppose une résistance à cette même classification biomédicale statistique à travers le regard qu'elles posent sur elle-même comme individu. Comme l'évoque Grenier (2009), la classification de la fragilité/dépendance, opérationnalisée à l'aide d'indicateurs biomédicaux et cliniques déterminant la prestation des services, fait abstraction des dimensions sociocognitive et émotionnelle qui y sont liées.

3 Désigne un déambulateur, soit un appareil pour faciliter la marche pour les personnes en perte de mobilité. 


\section{Discussion}

De toute évidence, les classifications de l'âge et de la fragilité/dépendance retenues apparaissent opérantes dans le rapport au vieillissement des répondantes. Plus encore, nous constatons qu'elles ont un effet «interactif », à savoir qu'elles expriment une interaction entre les individus et lesdites classifications parce que certaines d'entre elles ont une résonnance plus prégnante que d'autres: « Ce sont celles qui, une fois assimilées par les personnes et leur entourage, et une fois impliquées dans des institutions, modifient en retour la manière dont ces personnes s'éprouvent ellesmêmes. Ceci peut aller jusqu'à une modification de leurs sentiments et de leurs comportements, et cela en partie pour avoir été répertoriées de telle ou telle manière » (Hacking (2006 : 7). En l'occurrence, en dépit de l'apparition des représentations positives de la vieillesse, l'imaginaire social et les pratiques discursives entourant les femmes âgées restent plutôt péjoratifs (Charpentier et Billette, 2010; Perrig-Chiello, 2001). Par conséquent, les répondantes réfutent la catégorie "femmes aînées/âgées » non seulement parce qu'elle agît comme un facteur de stigmatisation (Mercier, 2010), mais aussi en raison de leurs " effets de façonnement» (Hacking, 2006) sur elles. Par exemple, l'étude de Grenier (2009) concernant les pratiques cliniques de la fragilité/dépendance au Québec indique que celles-ci tendent à renforcer le sentiment de fragilité des femmes et, a posteriori, consolident encore davantage ces classifications. En ce qui nous concerne, les répondantes interpellent ces classifications en mobilisant des représentations de soi positives et d'un rapport au vieillissement marqué par l'activité à tout âge, l'engagement et l'autonomie. Ce processus interactif entre représentations et classifications est aussi visible chez les répondantes qui adhèrent à la catégorie "femme aînée » avec une vision positive parce qu'elles la réinterprètent non pas en référence à l'âge (et à la démarcation vieille/jeune), mais en référence à la génération sociale (Mauger, 2000) et à la démarcation entre l'ancien et le nouveau. Ces propositions peuvent agir en retour sur ces mêmes classifications créant ainsi cet « effet de boucle » décrit par Hacking (2006). Ce dernier effet ne peut cependant pas être vérifié à partir du matériau, mais les changements survenus au cours des années dans les instruments de classification de la vieillesse témoignent néanmoins de leur perméabilité face aux énoncés et aux catégories sociologiques.

Par ailleurs, une interaction est aussi notable entre la classification de l'activité retenue et celles de l'âge et de la fragilité. L'activité - dans sa dimension du « faire »- est non seulement utilisée pour réfuter la vieillesse, mais aussi pour assurer cette « continuité de soi » dans l'avance en âge (Mercier, 2010). Lorsque le processus de fragilisation apparaît, ce dernier n'invalide pas l'idée d'être active pour plusieurs répondantes ; celle-ci est plutôt réorganisée et modifiée au regard des capacités physiques. Pour d'autres répondantes très âgées fragilisées, l'activité s'ancre davantage dans le quotidien : regarder la télévision, lire, et ce, afin de "rester dans la vie », de "s'intéresser à ce qui se passe dans l'actualité » et « dans le monde ». Autrement dit, lorsque la fragili- 
té s'approche graduellement du seuil de la dépendance, les «comparaisons descendantes » sont utilisées : l'accent est ainsi mis sur les activités que l'on peut encore accomplir et que d'autres - encore plus vieilles, malades ou fragilisées que soi - ne peuvent plus réaliser. Néanmoins, comme Boudiny (2012) l'évoque, ces activités de l'ordinaire expriment pourtant un engagement dans la vie. Ce passage du premier sens de l'activité (le faire) au second (l'engagement) expliquerait pourquoi les personnes très âgées s'estiment toujours actives, et ce, en dépit des classifications biomédicales et scientifiques qui, elles ne les évaluent plus comme des personnes actives. Finalement, lorsque la dépendance est manifeste, et que cette comparaison descente n'est plus possible, il s'agit alors de dissocier son identité et sa perception de soi du corps qui lâche prise. C'est dans ce moment de « face à soi » que plusieurs chercheront ainsi à négocier, sinon à réconcilier ce qu'ils sont et ont été avant de mourir.

\section{Conclusion}

«Le vieux ou la vieille », c'est toujours l'autre, comme l'ont souligné plusieurs autres écrits. L’autre «vieille » malade, dépendante et inactive. À cet égard, les répondantes ont intégré le discours dominant de l'activité dans le vieillir, notamment les « jeunes vieilles » qui font partie de cette première génération de femmes retraitées à être considérée statistiquement " actives », car elles ont occupé un emploi pendant la majeure partie de leur vie (Mc Donald, 2006). Pour d'autres, au parcours de vie traditionnel, notamment « les vieilles et les très vieilles » de notre échantillon, l'élargissement de la notion de l'activité dans les grilles et les politiques promouvant le vieillissement actif est importante, pour elles, mais aussi pour l'ensemble des femmes âgées, au regard des temporalités de vie différente des hommes et des femmes. La notion d'activité dans le vieillir n'est donc pas neutre en matière de genre, et ce, en raison de la prédominance de la trajectoire masculine en matière de travail-retraite (Foster et Walker, 2013). Considérant de plus que le vieillir actif reste articulé, dans certaines instances internationales, autour de l'employabilité, cette conception exclut non seulement les femmes n'ayant pas investi le marché de l'emploi, mais occulte l'ensemble du travail de reproduction et de care fourni par ces dernières (Ibid). Ainsi, puisque les logiques catégorielles de l'âge et du genre marquent la vie des individus ainsi que les instruments d'action publique, la statistique sociale et les différentes classifications qu'elle informe doivent prendre acte non seulement des dimensions socioémotives relatives aux expériences diversifiées de la vieillesse, mais aussi des rapports de pouvoir que ces catégorisations induisent entre et sur les différents groupes sociaux.

\section{Bibliographie}

Bickel J-F. (2007) : « Être actif dans le grand âge : un plus pour le bien-être? ». Retraite et société, $\mathrm{n}^{\circ} 51$, pp. 83-106. 
Boudiny K. (2012) : «Active ageing': from empty rhetoric to effective policy tool ». Ageing and Society, Vol. 1 n¹, pp.1-22.

Boudelais P. (1993): L'âge de la vieillesse. Histoire du vieillissement de la population. Paris : Éditions Odile Jacob.

Caradec V. (2007) : « L'épreuve du grand âge ». Retraite et société, n52, pp. 11-37.

Charpentier M. et Billette V. (2010): «Conjuguer 'vieillir' au féminin pluriel», In Charpentier M., Guberman N., Billette V., Lavoie J-P., Grenier A. et Olazabal G. (dir.). Vieillir au pluriel. Perspectives sociales, Presses de l'Université du Québec: Québec, pp. 75-88.

Foster L. et Walker A. (2013) : "Gender and active ageing in Europe. ». European Journal of Ageing, pp.1-8.

Grenier A. (2009) : «Femmes âgées et fragilité : leur résistance face aux pratiques du système de la santé et des services sociaux », In Charpentier C. et Quéniart A. (dir), Vieilles et après! Femmes, vieillissement et société. Montréal: Éditions du remue-ménage, pp. 249-269.

Guillemard A.-M. (1984) : « Jalons pour une sociologie des politiques sociales: Le cas de la politique française de la vieillesse ». Sociologie et société, vol 16, nº 2, pp.119-128.

Hacking I. (2006): Deux types de gens : les cibles mouvantes, Collège de France. http://www.college-de-france.fr/media/historique/UPL32427_types_de_gens.pdf

Katz S., Down T.D., Cash H.R., Grotz R.C. (1970): « Progress in the development of the index of ADL ». The Gerontologist, vol, 10, nº1, pp. 20-30

Hummel C. (2001) : «Représentations de la vieillesse chez les jeunes adultes et des octogénaires ». Gérontologie et société, nº98, 239-252.

Lavigne d'Épinay C., Spini D. (2008) : Les années fragiles. La vie au-delà de quatre-vingts ans. Québec : Les Presses de l'Université Laval.

Mauger G. (2009) : "Générations et rapports de générations », In. A. Quéniart, R. Hurtubise (dir.), L'intergénérationnel. Regards pluridisciplinaires, Rennes : Éditions de l'École nationale de Santé Publique, pp.17-36.

Mc Donald L. (2006): «La retraite selon le sexe: le bien- être des femmes de la 'nouvelle» retraite' ». In L.O. Stone, Les nouvelles frontières de recherche au sujet de la retraite, Ottawa, Statistiques Canada.

Mercier P. (2010) : « Souci de soi, souci de l'autre dans le processus du vieillissement ». DIALOGUES, Recherches sur le couple et la famille, $2^{\mathrm{e}}$ trimestre, pp. 39-51. 
Minkler M. (2007): « Two Critical gerontology: reflections for the $21^{\text {st }}$ century Critical perspectives on ageing societies ».. In M. Bernard, T. Scharf (dir.), Critical perspectives on ageing societies, Bristol : Policy Press, pp. 13-23.

Perrig-Chiello P. (2001) : « Images sexuées de la vieillesse : entre stéréotypes sociaux et autodéfinition $»$. Retraite et société, vol. 3 n 34, pp. 70-87.

Quéniart A., Charpentier M., Lebreton C. (2011): «La recherche féministe et les femmes aînées : une rencontre récente ». Labrys, Études féministes/ estudos feministas (juillet/décembre 2011 -janvier /juin 2012).

Ylieff M., De Lepeleire J., Buntix F. (2006): «La dépendance des personnes âgées. Définition, causes et conséquences", Vieillesse, dépendance et lieux de vie de l'Observatoire, $\mathrm{n}^{\circ} 44, \mathrm{pp} .1-6$. 


\section{Annexe 1- Classification de l'activité dans la vieillesse}

(Bickel, 2007, p.89-90)

\section{Être active/inactive dans la vieillesse}

\section{Liste de 16 activités regroupées dans sept domaines}

On peut les regrouper en sept grands domaines:

- les activités manuelles avec le jardinage, les travaux manuels (bricolage, couture...);

- les jeux (solitaires ou de société);

- les médias (radio et télévision);

- la lecture (journal d'une part, livres ou revues de l'autre);

- les activités extérieures: la fréquentation du café ou restaurant, la participation à des fêtes locales, les activités culturelles (aller au cinéma, au concert...), les excursions-voyages;

- les activités "physiques» (promenade d'une part, exercice physique ou pratique d'un sport de l'autre);

- la religion: la prière et la fréquentation de l'office religieux (qui inclut le fait de suivre l'office à la radio ou télévision). 


\section{Annexe 2 - La classification de la fragilité/dépendance/indépendance}

(Bickel, 2007, p.89)

\section{Fragilité}

Pour la rendre opérationnelle, la fragilité est approchée à l'aide d'une liste de dix-neuf atteintes appartenant à cinq dimensions de la santé:

- la mobilité (monter et descendre un escalier, se déplacer à l'extérieur du logement, parcourir 200 mètres à pied);

- les troubles physiques (dix items);

- les capacités sensorielles (lire un texte dans un journal, suivre une conversation à deux, suivre une conversation à plusieurs);

- la mémoire (avoir des troubles de mémoire);

- l'énergie (sentiment de fatigue et manque d'appétit).

À partir de là, trois statuts de santé sont définis:

- une personne est fragile (F) lorsqu'elle connaît des troubles portant sur au moins deux de ces cinq dimensions, tout en ne présentant aucune dépendance fonctionnelle, celle-ci étant définie par l'incapacité à accomplir seul une ou plusieurs des cinq activités de base de la vie quotidienne (AVQ) : voir Katz et al., 1970;

- I'état de dépendant (D) se définit par la présence de l'une ou l'autre de ces incapacités, distinct donc de celui de fragile;

- I'indépendance (I) désigne la situation dans laquelle se trouve la personne qui n'est concernée par aucune ou au maximum par une des cinq dimensions de santé, tout en ne souffrant bien sûr d'aucune incapacité fonctionnelle (pour une description plus détaillée de I'opérationnalisation de la fragilité, voir Armi, Guilley, 2004; Guilley et al., à paraître). 


\section{Annexe 3-Activity daily living (ADL)}

For each area of functioning listed below, check description that applies. (The word "assistance" means supervision, direction, or personal assistance.).

Bathing-either sponge bath; tub bath, or shower.

O Receives no assistance (gets in and out of tub by self if tub is usual means of bathing)

O Receives assistance in bathing only one part of the body (such as back or a leg)

O Receives assistance in bathing more than one part of the body (or not bathed)

Dressing - gets clothes from closets and drawers-including underclothes, outer garments and using fasteners (including braces if worn)

O Gets clothes and gets completely dressed without assistance

O Sets clothes and gets dressed without assistance except for assistance in tying shoes

O Receives assistance in getting clothes or in getting dressed, or stays partly or completely undressed

Toileting - going to the "toilet room" for bowel and urine elimination; cleaning self after elmination, and arranging

Clothes

O Goes to "toilet room," cleans self, and arranges clothes without assistance (may use object for support such as cane, walker, or wheelchair and may manage night bedpan or commode, emptying same in morning)

$\mathrm{O}$ Receives assistance in going to "toilet room" or in cleansing self or in arranging clothes after elimination or in use of

O Doesn't go to room termed "toilet" fo

\section{Transfer-}

O Moves in and out of bed as well as in and out of chair without assistance (may be using object for support such as cane or walker)

O Moves in and out of bed or chair with assistance

O Doesn't get out of bed

\section{Continence-}

O Controls urination and bowel movement completely by self 
O Has occasional "accidents'

O Supervision helps keep urine or bowel control; catheter

Table 2. Index of Independence in Activities of Daily Living.

The Index of Independence in Activities of Daily Living is based on an evaluation of the functional independence or dependence of patients in bathing, dressing, going to toilet, transferring, continence, and feeding. Specific definitions of functional independence and dependence appear below the index.

A-Independent in feeding, continence, transferring, going to toilet, dressing, and bathing.

$\mathrm{B}$-Independent in all but one of these functions.

C-Independent in all but bathing and one additional function.

D-Independent in all but bathing, dressing, and one additional function.

E-Independent in all but bathing, dressing, going to toilet, and one additional function.

F-Independent in all but bathing, dressing, going to toilet, transferring, and one additional function.

G-Dependent in all six functions.

Other-Dependent in at least two functions, but not classifiable as C, D, E, or F.

Source : Katz, S., Down, T.D., Cash, H.R., \& Grotz, R.C. (1970) Progress in the development of the index of ADL. The Gerontologist, 10(1), 20-30 